\title{
Um modelo computacional para auxiliar o ensino da temática de Terra como corpo cósmico
}

\author{
Nascimento, J. O. do ${ }^{1^{*}}$; Neide, I. G. ${ }^{2}$; Gonzatti, S. E. M. ${ }^{2}$; Moret, M. $A^{1,3}$ \\ 1 Departamento de Modelagem Computacional, Centro Universitário SENAI CIMATEC, Salvador, BA, Brasil. \\ 2 Centro de Ciências Exatas e Tecnológicas - CETEC, Centro Universitário UNIVATES, Lajeado, RS, Brasil. \\ 3 Universidade do Estado da Bahia, Salvador, BA, Brasil.
}

* e-mail: jeffersonascimento@gmail.com

\begin{abstract}
Resumo
Para facilitar o processo de ensino e de aprendizagem em Física, por meio da utilização de computadores, evitando-se aprender inúmeras linguagens de programações, foram desenvolvidos diversos softwares, dentre eles o Modellus. As nuances na sua utilização se concentram em processos que objetivam atividades que necessitam basicamente dos conceitos de Ciências, Matemática e estudos básicos de computação. Este artigo tem por objetivo apresentar um modelo computacional desenvolvido para utilização no Software Modellus e, auxiliar no ensino da temática de Terra como corpo cósmico. Esta abordagem à Terra significa, estudá-la por meio de sua forma, seu campo gravitacional, seus movimentos e os fenômenos astronômicos decorrentes desses movimentos e da configuração do sistema Sol-TerraLua, ou seja, a sua interação com outros astros cósmicos. Inicialmente, o método de construção por meio de um algoritmo é apresentado, e também, como deve ocorrer a sua implementação no software. As respectivas interações com as funções no Modellus são também indicadas. Ao fim, o modelo computacional do sistema Sol-Terra-Lua é apresentado de forma executável, auxiliando o processo de ensino de aprendizagem da Temática de Terra como um corpo cósmico.
\end{abstract}

Palavras-chave: Ensino de Física, Modelagem Computacional, Software Modellus, Terra como corpo cósmico.

\begin{abstract}
To facilitate the process of teaching and learning in physics, through the use of computers, avoiding to learn numerous programming languages, several software was developed, among them Modellus. The nuances in its use focus on processes that aim at activities that basically require the concepts of Science, Mathematics and basic computer studies. This article aims to present a computational model developed for use in Software Modellus and to assist in teaching the Earth thematic as a cosmic body. This approach to Earth means to study it through its form, its gravitational field, its movements and the astronomical phenomena arising from these movements and the configuration of the Sun-Earth-Moon system, that is, its interaction with other cosmic stars. Initially, the method of construction by means of an algorithm is presented, and also, how its implementation in the software should occur. The respective interactions with the functions in Modellus are also indicated. Finally, the computational model of the Sun-Earth-Moon system is presented in an executable form, aiding the learning process of the Earth Thematic as a cosmic body.
\end{abstract}

Keywords: Physics Teaching, Computer Modeling, Earth as a body cosmic.

\section{Introdução}

As últimas décadas são caracterizadas por uma revolução tecnológica, em que um dos principais reflexos é a evolução dinâmica da informática e dos computadores. Ao ocupar os diversos espaços da sociedade, no uso doméstico, influenciando o estilo de vida do homem, a tecnologia também se apresenta nas diversas relações de trabalho do setor industrial. Em relação ao campo educacional pode-se fazer presente pelo processo de aquisição do conhecimento. 
Os avanços tecnológicos resultam numa maior possibilidade do acesso à informação, elevando-se consideravelmente o número de pessoas que passam a ter este acesso, pois se vive em uma sociedade em que prevalecem a velocidade, o movimento, a imagem, o tempo e o espaço com uma nova conceituação [1]. Diante desta nova realidade, o uso do computador nos processos de ensino e de aprendizagem está cada vez mais comum, possibilitando assim, ampliar a utilização desta ferramenta e quando acrescentamos a importância do acesso à internet, de acordo com o autor supracitado, esta combinação se torna primordial para a construção, difusão e avanço do conhecimento.

Nesta lógica Nascimento et al. [2], ao utilizar as Tecnologias da Informação e da Comunicação (TIC) aplicadas ao ensino de Física, nos faz refletir sobre os processos de ensino e de aprendizagem atuais, em que ao encontrarmos alternativas para tornar as aulas de Física mais agradáveis e motivadoras. Assim, poderemos proporcionar uma melhor aprendizagem aos alunos, sendo este um desafio constante na prática docente. Araújo [3], Araújo [4] e Nascimento et al. [5] ratificam o uso de ferramentas tecnológicas no ensino de Física, como a modelagem computacional, por meio de propostas didáticas envolvendo 0 uso de computadores, em que softwares cada vez mais elaborados vêm sendo criados na tentativa de facilitar a construção do conhecimento por parte do estudante. Seguindo este pensamento, a fim de facilitar o processo de ensino e de aprendizagem e obter um ganho real de tempo em frente ao computador, foram desenvolvidos softwares como o Dynamic Modelling System, Stella, Easy Java Simulations e o Modellus, os quais concentravam a aprendizagem em atividades que necessitavam fundamentalmente dos conceitos de Ciências e Matemática [6]. Desta forma, nossa opção na presente pesquisa é apresentar um modelo computacional, para o estudo de fenômenos astronômicos, no Software Modellus.

Este trabalho está organizado em 6 seções. $\mathrm{Na}$ segunda, apresentaremos o Software Modellus. $\mathrm{Na}$ terceira os materiais e métodos utilizados para a construção do modelo computacional. Na quarta, os resultados e as discussões. Na quinta seção, as considerações finais e na sequência, os agradecimentos.

\section{O Software Modellus}

O Modellus conforme Teodoro, Vieira e Clérigo [7], é uma ferramenta de computador que fornece a possibilidade de alunos e professores poderem realizar experimentos conceituais de modelos matemáticos. As representações matemáticas podem ser funções, derivadas, taxa de variação, equações diferenciais e equações por meio de diferenças, bastando escrevê-las de forma simples e direta em uma caixa de texto chamada de "Modelo Matemático".
Representação Múltipla e Manipulação direta são características importantes no Modellus. A primeira consiste no fato de se poder criar, ver e interagir com representações analíticas, analógicas e gráficas de objetos matemáticos [3,4,5]. A segunda, manipulação direta, já citada anteriormente, significa que o usuário pode trabalhar diretamente com todos os tipos de objetos que aparecem na tela do computador, sem a necessidade de um conhecimento em uma determinada linguagem de programação, ou seja, os objetos podem ser manipulados de forma direta por obedecerem ao modelo matemático trabalhado [8]. O Modellus constitui uma ferramenta que também possibilita uma saída gráfica, pois, conforme o modelo computacional é executado, e os objetos adquirem movimentos, os gráficos são plotados instantaneamente. De forma concomitante a plotagem, acompanha o desenvolvimento das equações, que representam o modelo matemático que está sendo resolvido, conforme as condições iniciais atribuídas.

\section{Materiais e método}

A ferramenta utilizada para modelagem computacional foi o Software Modellus, que também pode ser encontrado para download em www.modellus.fct.unl.pt/, (website da Faculdade de Ciências e Tecnologia Universidade Nova de Lisboa), de onde o usuário poderá fazer download também de manuais e tutoriais em diversos idiomas. Encontram-se também inscrições para cursos, diversas versões do Modellus, artigos, brochuras em extensão de arquivo "pdf" e, exemplos de modelagem para Matemática e Física. A versão utilizada para o modelo computacional apresentado nesta pesquisa corresponde ao Software Modellus $X$, encontrado no site supracitado, que apresenta a seguinte tela inicial, conforme Figura 1:

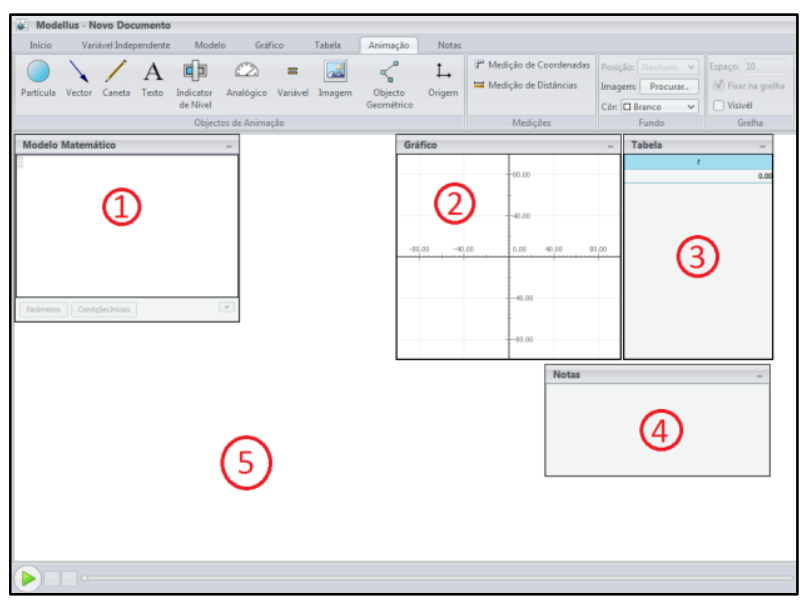

Figura 1: Software Modellus X. Fonte: Do software.

$\mathrm{Na}$ tela inicial apresentada na Figura 1 estão disponíveis os menus e submenus (como qualquer software) e algumas janelas que merecem nossa atenção. Com a numeração 1 a janela do modelo matemático, onde as equações devem ser dispostas de 
forma lógica e direta. A numeração 2 corresponde a tela cujos gráficos, que correspondem aos movimentos de animações que obedecem às equações matemáticas inseridas na tela do modelo matemático, são plotados. Na janela 3 são apresentados os valores resultantes dos cálculos realizados pelas equações inseridas na janela do modelo matemático. A janela 4 apresenta uma função de bloco de notas ou anotações de comentários. A região 5 indica a área de animação, onde os objetos serão inseridos e associados às equações matemáticas para o desenvolvimento do modelo computacional.

\section{Resultados e discussão}

Na presente pesquisa modelar o sistema SolTerra-Lua no Software Modellus basicamente significa construir um modelo computacional da equação da elipse associada ao movimento dos astros envolvidos com a possiblidade de translação dos eixos, conforme as equações abaixo:

$\frac{(x-h)^{2}}{a^{2}}+\frac{(y-k)^{2}}{b^{2}}=1$

A Eq. 1 e as equações a seguir utilizam a condição $2 a>2 b$, com o eixo $a$, estando na horizontal e o $b$, na vertical. Desta forma a Eq. 1 pode ser escrita da seguinte maneira:

$x=h+a \cdot \cos \theta$

e,

$y=k+b \cdot \operatorname{sen} \theta$

Os termos $h$ e $k$, representam os fatores de translação nas equações da elipse, na forma paramétrica. Para a construção do modelo computacional no Software Modellus, sugerimos os seguintes procedimentos abaixo:

1. Execute o Modellus e, no Menu Animação (Figura 2), escolha Partícula:

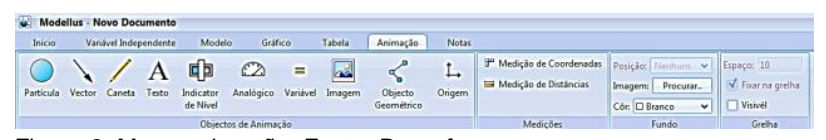

Figura 2: Menu animação. Fonte: Do software.

2. Clique na Área de Animação e insira o Objeto;

3. Retorne ao Menu Animação e altere o Objeto inserido pela que representa o Planeta Terra;

4. No Menu Animação, insira um Objeto Geométrico e altere o seu Tipo para Segmento de Reta;
5. Deixe o Objeto Geométrico com inclinação de aproximadamente $23,5^{\circ}$, em relação à direção horizontal;

6. No Menu Animação, insira o Objeto Origem;

7. Selecione o Objeto Geométrico e no menu Propriedades, faça a sua ligação - Ligar a com o Objeto Origem;

8. Junte a Terra o seu "eixo de inclinação", o Objeto Geométrico ligado ao Objeto Origem;

9. No Menu Animação, insira um novo Objeto Partícula, alterando sua Cor, para amarelo. Altere também o seu tamanho com o mouse, de forma a representar o nosso Sol, para o presente modelo;

Obs.: Inevitavelmente que o modelo Sol e Terra ficarão fora de proporção, para fins didáticos;

10. Aproxime Sol e Terra, deixe o periélio e afélio diferenciados em média de $3 \%$ de distância, no modelo;

11. Aproxime os Objetos na Área de Animação;

12. Para a janela Modelo Matemático apresentamos o seguinte algoritmo na forma de fluxograma na Figura 3, alicerçado e derivado das obras de Nascimento, Neide e Borragine [9] e Nascimento, Neide e Gonzatti [10]:

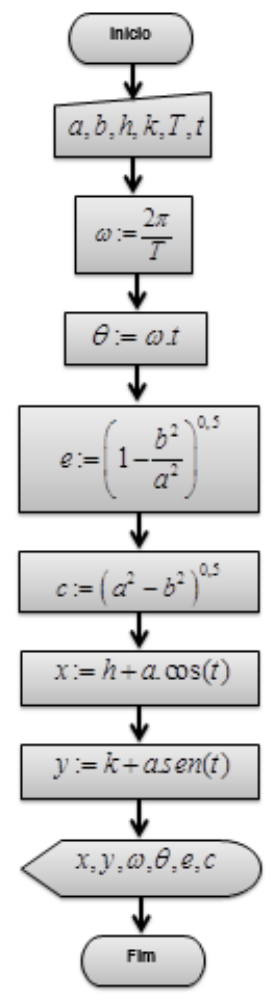


Figura 3: Algoritmo para o desenvolvimento do modelo computacional. Fonte: Do autor.

\section{Selecione o Objeto Terra;}

14. No Menu Propriedades (Figura 4), atribua como as coordenadas $x=x$ e $y=y$;

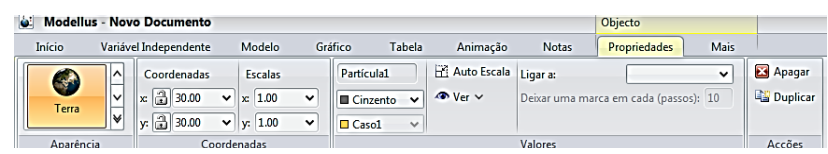

Figura 4: Menu Propriedades. Fonte: Do software.

15. As escolhas para $x$ e $y$, atribua uma unidade para cada. Selecione para visualização - Ver Trajetória e Eixos.

16. No Menu Mais, Selecione Ângulo e dentro de ângulo, teta.

17. Selecione o "Eixo inclinado da Terra", em sua Origem e na guia Propriedades, atribua como coordenadas, $\mathrm{x}$ e $\mathrm{y}$.

18. Nas posições correspondentes aos Solstícios e Equinócios, insira o Objeto Texto, acrescentando as informações correspondentes.

19. Clique no botão play e verifique o desenvolvimento da Modelagem.

O modelo computacional resultante para o estudo dos fenômenos astronômicos está indicado nas Figuras 4, 5, 6, 7 e 8:

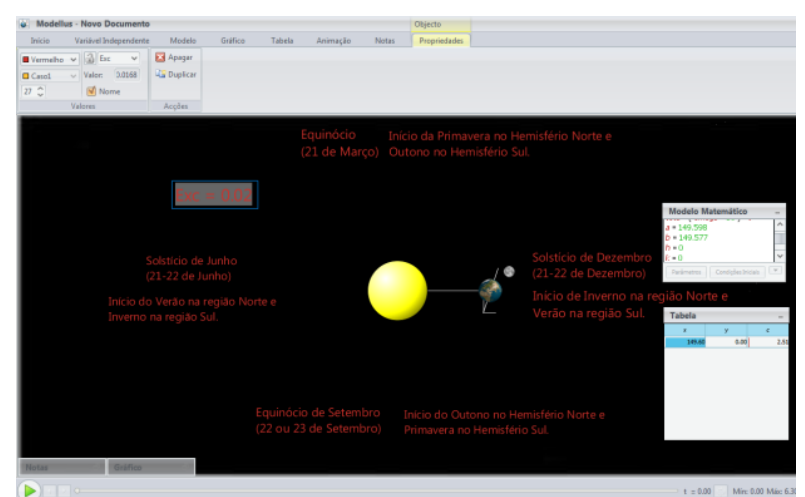

Figura 4: Inicio execução da modelagem - Solstício de dezembro. Fonte: Do software.

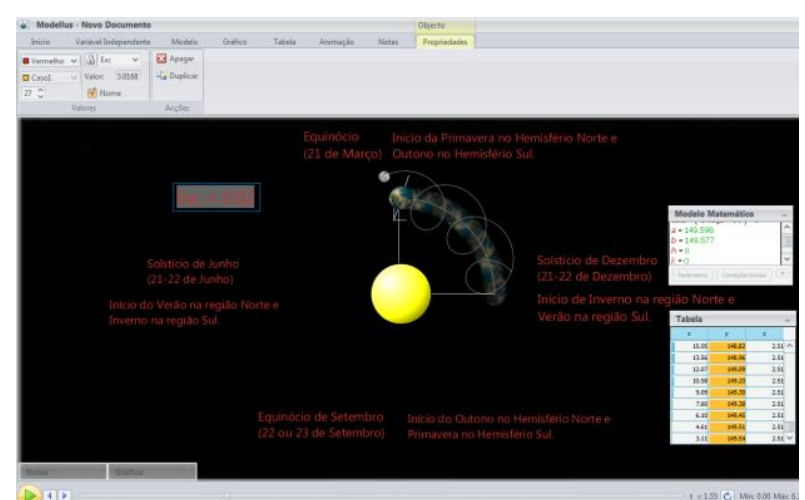

Figura 5: Modelo com ênfase na posição do Equinócio de Março. Fonte: Do software.

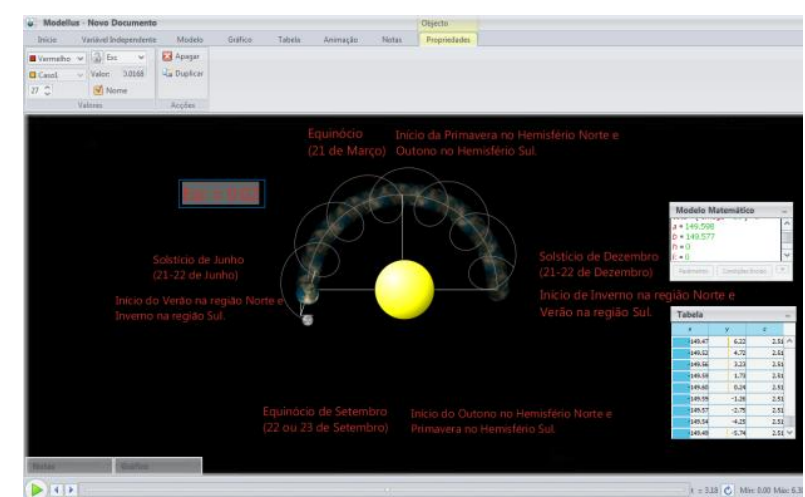

Figura 6: Modelo com ênfase na posição do Solstício de Junho. Fonte: Do software.

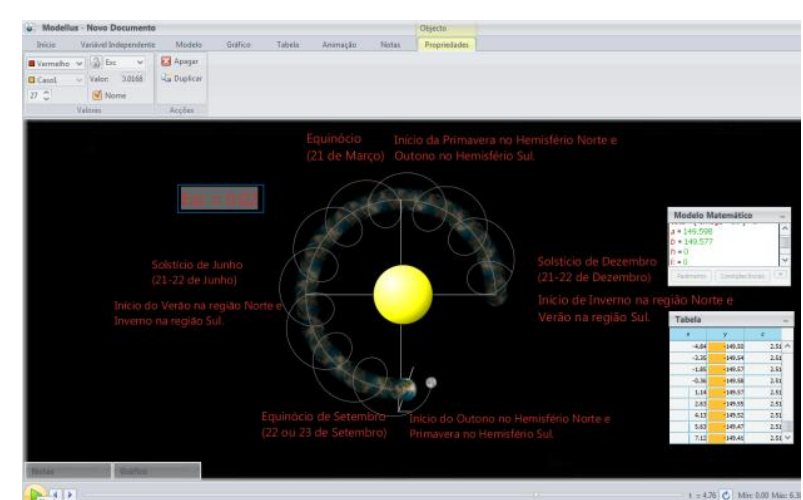

Figura 7: Modelo com ênfase na posição do Equinócio de Junho. Fonte: Do software.

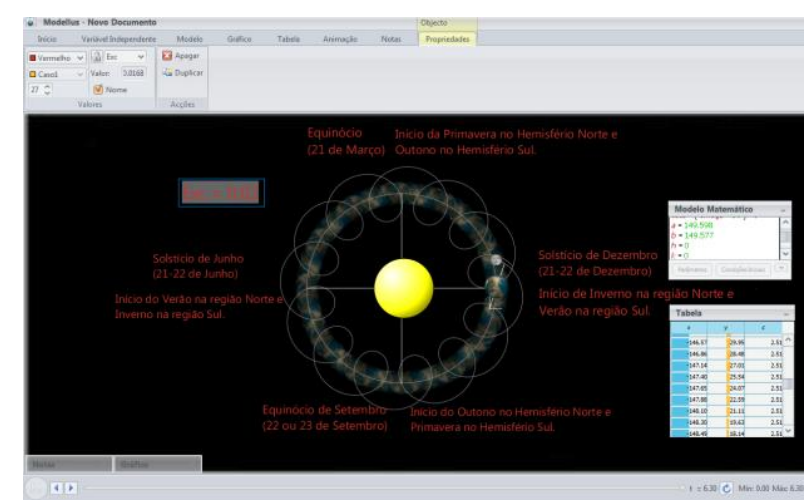

Figura 8: Final da execução modelagem para um período de um ano Solstício de dezembro. Fonte: Do software.

A título de exemplo, o resultado da aplicação do modelo computacional por meio de uma intervenção psicopedagógica é apresentado na pesquisa de Nascimento [11], Nascimento et al. [5] e Nascimento et al. [2].

\section{Considerações finais}

Os fenômenos astronômicos como os dias e noites, sucessão de anos e de estações, fases lunares são fenômenos comuns na nossa rotina e que estão associados à natureza cósmica da Terra, que envolve tanto sua forma, localização, quanto sua participação em interações gravitacionais. Desta forma ao considerarmos a Terra como um corpo cósmico, não significa caracterizá-la de forma perfeitamente esférica, de cor azul, quase sem nuvens, comumente mostrado nos diversos meios de comunicação, ou em modelos 
didáticos como globos terrestres e os mapas mundiais, presentes nos ambientes escolares, em que 0 encantamento visual é inevitável, bem como a dissociação do mundo real. Tratar a Terra como corpo cósmico, significa abordá-la por meio de sua forma, seu campo gravitacional, seus movimentos e os fenômenos astronômicos decorrentes desses movimentos e da configuração do sistema Terra-Sol-Lua, como por exemplo, dias, noites, estações do ano, fases e eclipses lunares, ou seja, a sua interação com outros astros cósmicos.

Desta forma e auxiliados das obras citadas nesta pesquisa de aplicação do modelo computacional apresentado neste artigo, por meio da apresentação do método de construção dele, esperamos que a modelagem computacional possa ser utilizada como ferramenta tecnológica para o Ensino de Física e áreas afins. O modelo tanto pode ser construído com aprendizes desde o início ou, entregue pronto para a utilização e estudo dos fenômenos astronômicos, havendo interseção e consonância com uma teoria de aprendizagem.

\section{Agradecimentos}

Jefferson Nascimento agrade à FAPESB devido ao apoio financeiro parcial por meio de sua bolsa de doutorado (BOL170/2015). Marcelo A. Moret agradece ao CNPq pelo suporte financeiro parcial oriundo de sua bolsa de Produtividade em Pesquisa (No. 304454/2014-1).

\section{Referências}

[1] NASCIMENTO, J. O. do; NEIDE, I. G.; GONZATTI, S. E. M. Modelagem e simulação computacional no ensino de Física: Uma proposta de estudo de caso com o PROEJA.. In: XXI Simpósio Nacional de Ensino de Física., 2015, Uberlândia/MG.. Anais do XXI SNEF.. 2015. v. 1.p. 1 - 8. Disponível em: < http://www.sbf1.sbfisica.org.br/eventos/snef/xxi/sys/resu mos/T0527-2.pdf>. Acesso em 07 ago. 2017.

[2] NASCIMENTO, J. O. do., NEIDE, I. G. , GONZATTI, S. E. M., MORET, M. A. . A modelagem e a simulação computacional como ferramentas tecnológicas no ensino de física. REVISTA SIGNOS - CENTRO UNIVERSITÁRIO UNIVATES, v. 38, p. 6282, n. 2017. Disponível em: < http://dx.doi.org/10.22410/issn.1983-

0378.v38i1a2017.1378>. Acesso em: 26 jul. 2017.

[3] ARAUJO, Ives Solano. Um Estudo sobre o desempenho de alunos de Física usuários da ferramenta computacional Modellus na interpretação de gráficos em cinemática. Porto Alegre: UFRGS, 2002. 111 p. Dissertação (Mestrado) Programa de Pós-Graduação em Ensino de Física, Instituto de Física, Universidade Federal do Rio Grande do Sul, Porto Alegre, 2002.
[4] ARAUJO, Ives Solano. Simulação e modelagem computa9cionais como recursos auxiliares no ensino de física geral. Tese (Doutorado em Ensino de Física) - Instituto de Física. Universidade Federal do Rio Grande do Sul, Porto Alegre, 2005.

[5] NASCIMENTO, J. O. do; NEIDE, I. G.; GONZATTI, S. E. M.; MORET, M. A.; "A modelagem computacional como ferramenta tecnológica no Ensino de Física", $p$. 115-120 . In: Anais do VII Encontro Científico de Física Aplicada [=Blucher Physics Proceedings, v.3 n.1]. São Paulo: Blucher, 2016. ISSN 2358-2359, DOI 10.5151/phypro-vii-efa-028. Disponível em:

http://www.proceedings.blucher.com.br/article-

[6] TEODORO, Vítor Duarte; NEVES, Rui Gomes. Mathematical modelling in science and mathematics education. Computer Physics Communications, Volume 182. Páginas 8-10. 2011. Disponível em: <http://modellus.fct.unl.pt/file.php/1/Teodoro_Neves_20 11_Mathematical_modelling_in_science_and_mathema tics education Computer Physics Communications.pd $\mathrm{f}>$. Acesso em: 03 mar de 2014

[7] TEODORO, V. D.; VIEIRA, J. P.; CLÉRIGO, F. C. Modellus, interactive modelling with mathematics. San Diego: Knowledge Revolution, 1997.

[8] TEODORO, V. D. From formulae to conceptual experiments: interactive modelling in the physical sciences and in mathematics. In: international colos conference new network-based media in education. Maribor, Slovenia: 1998. Disponível em: <http://www.if.ufrgs.br/tex/fis01043/textos/VDTeodoro1 998.pdf>. Acesso em: 20 jan. 2014.

[9] NASCIMENTO, J. O. do; NEIDE, I. G.; BORRAGINI, L. F. Modelagem computacional com o Software Modellus: Estudando as estações do ano.. In: XV EPEF - Encontro de Pesquisa em Ensino de Física, 2014, São Sebastião-Maresias/SP. Anais do XV EPEF. 2014. v. 1.p. 1- 8. Disponível em: < http://www.sbf1.sbfisica.org.br/eventos/epef/xv/sys/resu mos/T0355-1.pdf>. Acesso em 05 jul.. 2017.

[10] NASCIMENTO, J. O. do; NEIDE, I. G; GONZATTI, S. E. M. Modelagem e simulação computacional no ensino de Física: Uma proposta de estudo de caso com o PROEJA. In: XXI Simpósio Nacional de Ensino de Física, 2015, Uberlândia/MG. Anais do XXI SNEF, 2015. v. 1. p. 1-8. Disponvíel em: < http://www.sbf1.sbfisica.org.br/eventos/snef/xxi/sys/resu mos/T0527-2.pdf>. Acesso em: 04 mar. 2015

[11] NASCIMENTO, Jefferson Oliveira do. O Ensino de Física por meio de ferramentas tecnológicas: um estudo de caso com o PROEJA. 2015. $231 \mathrm{f}$. Dissertação (Mestrado em Ensino de Ciências Exatas) - Centro Universitário UNIVATES, Lajeado, 25 set. 2015. 
